\title{
SEQUÍAS Y PRENSA REGIONAL EN LA CUENCA DEL RÍO MUGA (GIRONA)
}

\author{
Carles Bayés Bruñol ${ }^{*}$, Anna Ribas Palom* y David Saurí Pujol ${ }^{\star *}$ \\ *Universitat de Girona. Unidad de Geografía. Plaça Ferrater Mora, 1. 17071 Girona \\ **Universitat Autònoma de Barcelona. Departamento de Geografía. 08193 Bellaterra (Barcelona) \\ carles.bayes@udg.es, anna.ribas@udg.es, david.sauri@uab.es
}

\begin{abstract}
Resumen: La sucesión casi permanente de las situaciones críticas de escasez hídrica en la cuenca del río Muga ponen de manifiesto la consideración de la sequía como un riesgo natural de creciente responsabilidad social. En este artículo se plantea un reconocimiento territorial de las sequías en esta cuenca a partir del apoyo instrumental de la prensa regional con el objetivo de delimitar el alcance espacial y temporal de las crisis hídricas desde el año 1950 hasta el 2001. También se utiliza la prensa para identificar las situaciones de conflicto entre disponibilidad y usos del agua, calidad del agua y de los ecosistemas fluviales e interpretar las soluciones de planificación y gestión del recurso llevadas a cabo con el objetivo de resolver estos conflictos.
\end{abstract}

Palabras clave: Sequía, escasez hídrica, prensa regional, río Muga, Cataluña, gestión del agua

\begin{abstract}
The almost continuous succesion of critical periods of water shortages in the La Muga river basin supports the definition of droughts as natural hazards increasingly caused by human activities. In this paper we offer a territorial survey of droughts in the La Muga basin drawn from local media sources and with the objective of assessing the spatial and temporal extent of water crises from 1950 to 2001. We also use the local press to identify conflict situations regarding water availability and use, water quality, and ecosystem health, and to interpret management options adressed at solving these conflicts.
\end{abstract}

Key words: Drought, water shortages, regional press, Muga river, Catalonia, water management

\footnotetext{
* Recibido: 6-02-2003. Aceptado: 3-07-2003.
} 


\section{Introducción}

El análisis del tratamiento que los medios de comunicación otorgan a los episodios de sequía resulta ser un instrumento muy idóneo para conocer con toda fidelidad el grado de conmoción que genera la sequía en la sociedad, incluyendo no sólo los impactos objetivos, sino también la valoración que de ellos hace la sociedad (Pita López, 1985). Si los pormenores del clima son muchas veces noticia, es porque tienen repercusiones de diversa índole en la vida del ser humano. La sequía o el exceso de agua, los aguaceros intempestivos, las heladas "fuera de época", etc. son, por ejemplo, fuente constante de preocupación para los campesinos y los labradores (Ruiz Urrestarazu, 1998). La trascendencia de un episodio de sequía depende en gran medida de la percepción que la sociedad tenga de él que puede depender mucho más de los mensajes de los medios de comunicación (prensa, radio y televisión) que de hechos meramente objetivos como las reservas de agua en los embalses, las precipitaciones registradas, o las pérdidas en las cosechas (Llamas, 2000).

Este artículo tiene un doble objetivo. Por una parte, reconocer las características espaciales y temporales de las sequías pluviométricas en la cuenca del río Muga $^{1}$ (Alt Empordà, Girona) (ver mapa 1) durante la segunda mitad del siglo XX $y$, por otra, ahondar en las posibilidades que ofrece la prensa regional para el análisis y la mejora de la gestión de las sequías en la cuenca, utilizando como fuente de información básica la prensa regional. En el presente trabajo se han consultado las noticias relacionadas con la sequía publicadas durante el período 1950-2001 en L'Empordà, un semanario de la comarca del Alt Empordà que viene publicándose sin interrupción desde septiembre de 1942. En este caso fueron consultados más de 2.400 ejemplares lo que dio como resultado 422 noticias directamente relacionadas con la problemática de la escasez hídrica en la cuenca de estudio. Una limitación a tener en cuenta es el hecho de tratarse de un medio de publicación de periodicidad semanal (lo que dificulta la actualización diaria de la información en cuestión), pero por contra tiene la ventaja de dedicar mayor atención a las noticias más destacadas de cada semana, a fin de satisfacer las necesidades informativas de la sociedad especialmente interesada por los acontecimientos de actualidad en la comarca.

La recopilación, sistematización y tratamiento de la información periodística permite multitud de análisis e interpretaciones (Silva Costa et al., 2002). En el presente trabajo nos hemos propuesto profundizar en dos de ellas. En primer lugar, analizar y valorar el nivel de conexión temporal y espacial entre el evento climático y los impactos territoriales de las sequías en la cuenca de estudio, a partir de poner en relación el comportamiento de la precipitación y el estado de las reservas hídricas en la cuenca con la frecuencia de noticias relativas a los episodios de escasez hídrica. 
En segundo lugar, identificar las situaciones de conflicto entre disponibilidad y usos del agua, calidad del agua y estado de los ecosistemas fluviales, que podría ayudar a interpretar las futuras actuaciones de planificación y gestión del recurso en esta región notablemente vulnerable a la escasez del agua. Para ello se ha realizado un análisis del contenido de los noticias publicadas a partir de su agrupación por bloques temáticos.

El artículo se estructura en cuatro apartados. En un primer apartado se identifican los periodos climáticos secos que se han dado en la cuenca del río Muga en el periodo objeto de estudio. A continuación se analiza el nivel de conexión temporal (apartado 3) y espacial (apartado 4) entre el evento climático (periodos climáticos secos) y los impactos territoriales ocasionados por la falta de agua (número de noticias publicadas). Por último, y a partir del análisis temático de las noticias, se determinan los principales problemas existentes en cada momento en relación a la falta de agua y las diversas soluciones adoptadas para solucionar estos problemas.

\section{La identificación de períodos secos a partir del análisis pluviométrico}

La definición de un período de sequía desde un punto de vista objetivo acostumbra a identificarse con los períodos marcados por reducciones significativas de la precipitación (sequía pluviométrica) respecto a la media del período de tiempo seleccionado (en nuestro caso 1950-2001), si bien comparte responsabilidades con el comportamiento de otros parámetros meteorológicos (altas temperaturas, elevados niveles de evapotranspiración, intensidad de los vientos, etc.). En este trabajo se han utilizado dos métodos para la identificación de los periodos de sequía pluviométrica en la cuenca del río Muga. En primer lugar se han aplicado los índices de frecuencia, calculados a partir de los valores de los quintiles de la serie de precipitación, siendo estos valores los puntos que dividen la distribución con base en el porcentaje acumulado de casos $^{2}$. Es un método creado por Gibbs y Maher el año 1967 (Almarza, 2000), que identifica el carácter pluviométrico (seco o húmedo) de los distintos años a partir de una descripción empírica (en base a una serie de datos observados) y estática (sin vinculación con resultados anuales precedentes) de las experiencias anuales de la precipitación registrada durante la segunda mitad del siglo XX en distintas estaciones meteorológicas de la cuenca de estudio. Los resultados de la aplicación de este método se recogen en la tabla 1. En la tabla 2 se precisan los umbrales de precipitación que determinan los caracteres pluviométricos para las respectivas estaciones meteorológicas seleccionadas (ver mapa 1) 
Tabla 1. Carácter pluviométrico de los años de estudio (1950-2001) en la cuenca del río Muga.

\begin{tabular}{|c|c|c|c|c|c|c|c|c|c|c|c|c|c|}
\hline & 1 & 2 & 3 & 4 & 5 & 6 & & 1 & 2 & 3 & 4 & 5 & 6 \\
\hline Altitud $(\mathrm{m})$ & 193 & 168 & 110 & 61 & 39 & 17 & Altitud (m) & 193 & 168 & 110 & 61 & 39 & 17 \\
\hline 1950 & MS & & & $S$ & $\mathrm{~N}$ & & 1976 & $S$ & $\mathrm{~L}$ & & $*$ & $\mathrm{~S}$ & $\mathrm{~L}$ \\
\hline 1951 & ML & & & ML & ML & & 1977 & S & ML & & * & ML & ML \\
\hline 1952 & MS & & & MS & MS & MS & 1978 & MS & MS & & $*$ & S & $\mathrm{N}$ \\
\hline 1953 & $\mathrm{~L}$ & & & $\mathrm{~N}$ & $S$ & $\mathrm{~N}$ & 1979 & $\mathrm{~N}$ & L & & $*$ & $\mathrm{~N}$ & $\mathrm{~N}$ \\
\hline 1954 & $\mathrm{~N}$ & & & $S$ & MS & MS & 1980 & $\mathrm{~L}$ & N & & * & MS & S \\
\hline 1955 & $\mathrm{ML}$ & & & $\mathrm{L}$. & $\mathrm{N}$ & $\mathrm{L}$ & 1981 & N & S & & $*$ & $\mathrm{~L}$ & $\mathrm{~N}$ \\
\hline 1956 & L & & & $\mathrm{S}$ & MS & MS & 1982 & ML & ML & & * & ML & L \\
\hline 1957 & ML & & & $\mathrm{L}$ & $\mathrm{L}$ & $\mathrm{L}$ & 1983 & MS & S & & * & MS & MS \\
\hline 1958 & $\mathrm{~N}$ & & & $N$ & $\mathrm{~S}$ & $S$ & 1984 & $N$ & $\mathrm{~N}$ & & * & $\mathrm{N}$ & $\mathrm{L}$ \\
\hline 1959 & $*$ & & & ML & L & ML & 1985 & MS & MS & & * & S & $\mathrm{S}$ \\
\hline 1960 & * & & & $\mathrm{N}$ & $S$ & $N$ & 1986 & L & $\mathrm{L}$ & & $\mathrm{L}$ & $\mathrm{N}$ & $\mathrm{N}$ \\
\hline 1961 & * & & & MS & MS & $S$ & 1987 & $\mathrm{~L}$ & $\mathrm{~L}$ & & ML & $\mathrm{ML}$ & ML \\
\hline 1962 & $*$ & & & $\mathrm{~L}$ & ML & $\mathrm{L}$ & 1988 & $\mathrm{~N}$ & $\mathrm{~N}$ & & $S$ & $\mathrm{~N}$ & MS \\
\hline 1963 & * & & & ML & ML & ML & 1989 & $\mathrm{~L}$ & $\mathrm{~L}$ & $\mathrm{~L}$ & $\mathrm{~N}$ & $\mathrm{~N}$ & S \\
\hline 1964 & * & & & $\mathrm{L}$ & $\mathrm{L}$ & $\mathrm{N}$ & 1990 & S & $S$ & MS & MS & $S$ & MS \\
\hline 1965 & * & & & ML & ML & ML & 1991 & ML & $\mathrm{ML}$ & ML & $\mathrm{N}$ & L & S \\
\hline 1966 & * & & & MS & MS & MS & 1992 & $\mathrm{ML}$ & $\mathrm{ML}$ & $\mathrm{ML}$ & ML & $\mathrm{ML}$ & $\mathrm{L}$ \\
\hline 1967 & * & & & $S$ & S & $\mathrm{S}$ & 1993 & I & $\mathrm{L}$ & L & ML & ML & ML \\
\hline 1968 & * & & & $\mathrm{N}$ & $\mathrm{N}$ & $\mathrm{N}$ & 1994 & S & $\mathrm{N}$ & $\mathrm{N}$ & $\mathrm{L}$ & $\mathrm{L}$ & ML \\
\hline 1969 & ML & & & ML & ML & ML & 1995 & $\mathrm{~N}$ & $\mathrm{~N}$ & $\mathrm{~N}$ & $S$ & $\mathrm{~N}$ & $S$ \\
\hline 1970 & $S$ & & & MS & MS & MS & 1996 & ML & ML & ML & ML & ML & ML \\
\hline 1971 & $\mathrm{ML}$ & & & ML & ML & ML & 1997 & $\mathrm{~N}$ & $N$ & $\mathrm{~L}$ & $\mathrm{~N}$ & $\mathrm{~N}$ & $\mathrm{~N}$ \\
\hline 1972 & ML & $\mathrm{ML}$ & & * & $\mathrm{L}$ & $\mathrm{L}$ & 1998 & MS & MS & MS & MS & $\mathrm{L}$ & MS \\
\hline 1973 & MS & MS & & * & MS & MS & 1999 & $\mathrm{~N}$ & $S$ & $\mathrm{~N}$ & $\mathrm{~L}$ & $\mathrm{~L}$ & $\mathrm{~N}$ \\
\hline 1974 & $S$ & MS & & $*$ & MS & $S$ & 2000 & $\mathrm{~L}$ & $S$ & $S$ & $\mathrm{~S}$ & $\mathrm{~L}$ & MS \\
\hline 1975 & MS & $S$ & & $*$ & S & $\mathrm{N}$ & 2001 & MS & MS & $S$ & MS & MS & MS \\
\hline
\end{tabular}

MS= Muy Seco; S= Seco; N= Normal; L= Lluvioso; ML= Muy Lluvioso

Estaciones meteorológicas, por orden topográfico: 1. Darnius; 2. Embalse Boadella-Darnius; 3. La Jonquera; 4. Peralada; 5. Figueres; 6. Castelló d’Empúries; * Interrupción de la serie pluviométrica Fuente: Elaboración propia.

Tabla 2. Determinación de los umbrales pluviométricos anuales $(\mathrm{mm})$ en la cuenca del río Muga.

\begin{tabular}{|l|c|c|c|c|c|c|c|}
\hline & Umbral (mm) & 1 & 2 & 3 & 4 & 5 & 6 \\
\hline Año muy seco & Lluvia< & 660 & 585 & 675 & 529 & 442 & 517 \\
Año seco & Lluvia entre & 660 y 772 & 585 y 688 & 675 y 804 & 529 y 593 & 442 y 513 & 517 y 590 \\
Año normal & Lluvia entre & 772 y 928 & 688 y 772 & 804 y 927 & 593 y 635 & 513 y 603 & 590 y 673 \\
Año lluvioso & Lluvia entre & 928 y 1064 & 772 y 884 & 927 y 1225 & 635 y 772 & 603 y 768 & 673 y 843 \\
Año muy lluvioso & Lluvia > & 1064 & 884 & 1225 & 772 & 768 & 843 \\
\hline
\end{tabular}

Estaciones meteorológicas, por orden topográfico: 1.Darnius; 2. Embalse de Boadella-Darnius; 3. La Jonquera; 4. Peralada; 5. Figueres; 6. Castelló d'Empúries

Fuente: Elaboración propia. 


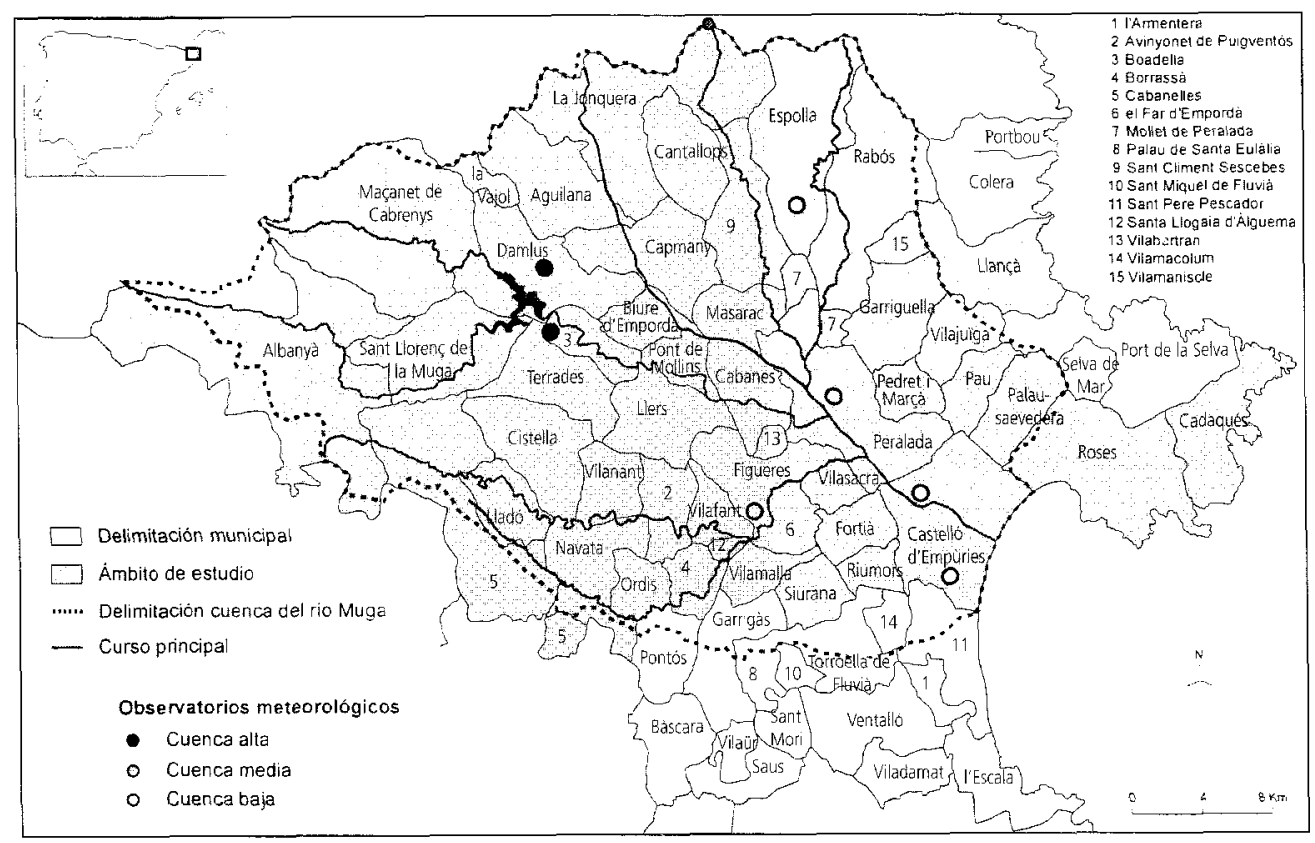

Mapa 1. Ámbito de estudio y localización de las estaciones meteorológicas de análisis.

En los años cincuenta se alternan años muy secos con años normales y años lluviosos en toda la cuenca, si bien es de destacar que las lluvias fueron especialmente abundantes en la cuenca alta durante la segunda mitad de la década (años 1955, 1956 y 1957). Entre la segunda mitad de los años sesenta y la primera de los setenta se extiende un periodo relativamente seco en toda la cuenca que se ve alterado por dos años consecutivos extremadamente lluviosos (1971 y 1972), precedidos por un 1969 también muy húmedo. Les sucede una secuencia muy seca entre 1973 y 1975, situación ésta representativa de la alta variabilidad interanual de la precipitación en los dominios mediterráneos. Los años ochenta responden a un ritmo muy variable en el comportamiento pluviométrico sucediéndose continuamente años de distinto carácter. A finales de los años noventa y durante el 2000 y 2001, la falta de precipitaciones fue acusada en toda la cuenca, especialmente en la cuenca alta, donde los notables déficits de precipitaciones de los años 1998 y 2001 sólo se ven interrumpidos por cortas transiciones húmedas que resultaron insuficientes para compensar los déficits acumulados anteriormente.

Sin embargo, la irregular distribución intranual de la precipitación exige un análisis temporal más acotado y obliga a trabajar a una escala mensual para entender de forma rigurosa las particularidades de las anomalías pluviométricas. El Índice 
Estandarizado de Sequía Pluviométrica, propuesto por M.F. Pita López el año 1987, y revisado por la misma autora en el 2000 , precisa las acumulaciones de los déficits pluviométricos mensuales, cálculo éste muy adecuado para interpretar la frecuencia, la intensidad y la duración del evento natural y su traducción sobre el territorio. El principal objetivo es delimitar el inicio y el cese de la sequía estrictamente pluviométrica a partir de la acumulación de los déficits de lluvia y la marginación de los excesos acumulados positivos.

En la aplicación del Índice Estandarizado de Sequía Pluviométrica se han precisado mensualmente los límites temporales de las secuencias secas. Se han considerado las secuencias secas de duración superior a los doce meses, que son las que realmente interesan por sus repercusiones en la vida social, económica y ambiental de la cuenca, ampliamente organizada a partir de las aguas reguladas por el embalse de Boadella-Darnius, infraestructura de regulación de régimen hiperanual (por una renovación de las aguas reguladas de ciclo escasamente superior a un año).

En la tabla 3 se especifican los límites mensuales de los periodos secos en las estaciones de Darnius (cuenca alta), Figueres (cuenca media) y Castelló d'Empúries (cuenca baja). En Darnius, el $51 \%$ de los meses de la serie considerada están implicados en las secuencias secas superiores al año, mientras que el peso de los meses posteriores a los doce primeros meses del periodo seco no supera el 18\% del total. Para el caso de Figueres, el 49\% de los meses analizados están implicados en las secuencias secas, reduciéndose hasta el $28 \%$ cuando sólo se consideran los meses posteriores a los doce primeros. En Castelló d'Empúries participan de esta tendencia el 37\% del total de meses de las serie de referencia. Si no se consideran los doce primeros meses del periodo seco se obtiene un porcentaje equivalente al $21 \%$ del total de la serie.

Los resultados de la aplicación de este segundo método son indicativos del carácter acumulativo de las deficiencias en la precipitación y de la evolución de sus repercusiones sobre el territorio. De esta manera se precisa el enlace cronológico entre el evento físico y las implicaciones socioterritoriales derivadas de la escasez de agua y, además, puede interpretarse la responsabilidad del componente físico en la aparición y caracterización del fenómeno de la sequía. Desde un punto de vista estrictamente climático, se dibuja una apreciable disparidad temporal y espacial de las precipitaciones en la cuenca. En la cuenca alta se dan intensidades medias de los episodios con sequía pluviométrica inferiores a los de la cuenca media y baja, distribuidas en secuencias generalmente más cortas y frecuentes que superan en menor número de casos el umbral de los doce meses. Aún así, y en repetidas ocasiones, la reducción mensual absoluta de la precipitación respecto a los valores habituales es más elevada en la cuenca alta como respuesta a episodios locales muy secos, que van a afectar las disponibilidades hidrológicas de la cuenca. 
Esta irregularidad espacial de la pluviometría debe valorarse como un recurso de compensación territorial a tener en cuenta en la planificación hídrica de la cuenca. Así por ejemplo, una descentralización de las fuentes básicas de suministro de agua, hoy en día dependientes de unas infraestructuras localizadas en unos pocos sectores de la cuenca (especialmente el embalse Boadella-Darnius) podría proporcionar una mayor flexibilización de la oferta de agua en la cuenca.

Tabla 3. Secuencias secas superiores al año en la cuenca del río Muga (1950-2001).

\begin{tabular}{|c|c|c|c|c|c|}
\hline Secuencias secas & \multicolumn{2}{|c|}{ Duración (meses) } & \multicolumn{2}{|c|}{ Intensidad máxima } & $\begin{array}{c}\text { Meses secos } \\
>1 \text { año seco }\end{array}$ \\
\hline \multicolumn{6}{|l|}{ Darnius (1969-2001) } \\
\hline Noviembre 1969-Febrero 1971 & & 16 & & $-1,86$ & 4 \\
\hline Octubre 1972- Junio 1977 & & 57 & & $-2,18$ & 45 \\
\hline Agosto 1977 - Diciembre 1978 & & 17 & & $-1,86$ & 5 \\
\hline Febrero 1979- Enero 1980 & & 12 & & $-1,55$ & 0 \\
\hline Enero 1983- Octubre 1984 & & 22 & & $-1,84$ & 10 \\
\hline Diciembre 1984- Enero 1986 & & 14 & & $-1,56$ & 2 \\
\hline Mayo 1986- Junio 1987 & & 14 & & $-1,70$ & 2 \\
\hline Julio 1988- Octubre 1989 & & 16 & & $-1,41$ & 4 \\
\hline Noviembre 1994- Noviembre 1995 & & 13 & & $-1,54$ & 1 \\
\hline Enero 1998- Diciembre 1998 & & 12 & & $-1,12$ & 0 \\
\hline \multirow{2}{*}{ Diciembre 1999- Noviembre 2000} & & 12 & & $-1,49$ & 0 \\
\hline & Total & 205 & Media, & $-1,65$ & 73 \\
\hline \multicolumn{6}{|l|}{ Figueres (1950-2001) } \\
\hline Noviembre 1951- Noviembre 1958 & & 85 & & $-2,25$ & 73 \\
\hline Noviembre 1959- Septiembre 1962 & & 35 & & $-2,46$ & 23 \\
\hline Noviembre 1965- Enero 1967 & & 15 & & $-1,03$ & 3 \\
\hline Abril 1967- Noviembre 1968 & & 20 & & $-1,71$ & 8 \\
\hline Diciembre 1969-Junio 1971 & & 19 & & $-1,58$ & 7 \\
\hline Febrero 1973- Abril 1977 & & 51 & & $-1,78$ & 39 \\
\hline Febrero 1979- Mayo 1980 & & 16 & & $-1,27$ & 4 \\
\hline Abril 1983-Abril 1984 & & 13 & & $-1,55$ & 1 \\
\hline \multirow[t]{2}{*}{ Junio 1988- Junio 1990} & & 25 & & $-2,86$ & 13 \\
\hline & Total & 339 & Media, & $-1,83$ & 171 \\
\hline \multicolumn{6}{|l|}{ Castelló d'Empúries (1952-2001) } \\
\hline Abril 1952- Febrero 1954 & & 23 & & $-1,74$ & 11 \\
\hline Agosto 1954- Julio 1955 & & 12 & & $-1,59$ & 0 \\
\hline Noviembre 1965- Octubre 1968 & & 36 & & $-1,07$ & 24 \\
\hline Noviembre 1972- Agosto 1975 & & 34 & & $-2,21$ & 22 \\
\hline Febrero 1979- Abril 1980 & & 15 & & $-1,43$ & 3 \\
\hline Diciembre 1982- Abril 1985 & & 29 & & $-1,71$ & 17 \\
\hline Junio 1988- Diciembre 1991 & & 43 & & $-2,48$ & 31 \\
\hline Noviembre 1994- Noviembre 1995 & & 13 & & $-1,33$ & 1 \\
\hline Mayo 1998- Agosto 1999 & & 16 & & $-1,64$ & 4 \\
\hline Diciembre 1999-Diciembre 2000 & & 13 & & $-2,60$ & 1 \\
\hline$\cdot$ & Total & 234 & Media, & $-1,78$ & 114 \\
\hline
\end{tabular}

Fuente: Elaboración propia. 


\section{Conexión temporal entre el evento climático y los impactos territoriales de las sequías}

El análisis y la gestión de las sequías requiere caracterizar la complejidad de un riesgo singular entre la amplia diversidad de riesgos naturales existentes. En las sequías, la conexión espacio-temporal entre el evento climático y los impactos territoriales es mucho menor que la que se registra en los restantes tipos de desastres, lo que ha llevado a decir que "la independencia naturaleza-sociedad es mucho más marcada en el caso de las sequías. Y, sin embargo, en el análisis de las sequías la conexión entre el evento y los impactos es mucho más necesaria que en ningún otro caso. La sequía carece de identidad si la desconectamos de los impactos: son éstos los únicos capaces de fijar el umbral de sequía en la escala variable de las precipitaciones" (Pita López, 1990: 25).

La correspondencia entre el comportamiento de la precipitación y la frecuencia de noticias relativas a los episodios de escasez hídrica en el territorio permiten reconstruir la cronología de las repercusiones sociales, económicas y ambientales de la sequía en la cuenca y, en este mismo caso, considerar el grado de responsabilidad de los aspectos físicos en el acaecimiento de los periodos con déficit hídrico.

Ya se ha comentado anteriormente que la resolución anual de los registros pluviométricos no es la más adecuada para interpretar unos resultados propios de entornos climáticos caracterizados por la elevada variabilidad y disparidad pluviométricas (Martín Vide, 1990). Aún así, y en general, se observa una relación indirectamente' proporcional entre el número de noticias publicadas anualmente y el total de precipitación media anual registrada ${ }^{3}$. Sin embargo, no son extraños los años lluviosos con implicaciones severas sobre el territorio por una reducción significativa de los registros pluviométricos en determinados meses del año. En la cuenca cabe destacar el año 1994, sometido a un déficit inicial de precipitación muy preocupante, responsable de importantes consecuencias territoriales y ambientales en la cuenca, y posteriormente compensado con generosas aportaciones de lluvia que atenuaron la disminución experimentada por las reservas hídricas y cambiaron el carácter pluviométrico de este año. Lo mismo ocurre en el año 1989, cuando los meses de octubre, noviembre y diciembre acumulan la mitad de la precipitación total anual, precedida por un episodio seco notablemente intenso; o también en el año 1987, cuando entre enero y septiembre suman poco más del $50 \%$ de la precipitación total, y, además, se acentúan los episodios de contaminación fluvial que repercuten negativamente en la calidad del agua disponible. La consideración de estas anomalías distorsiona significativamente la lectura de la figura 1. 


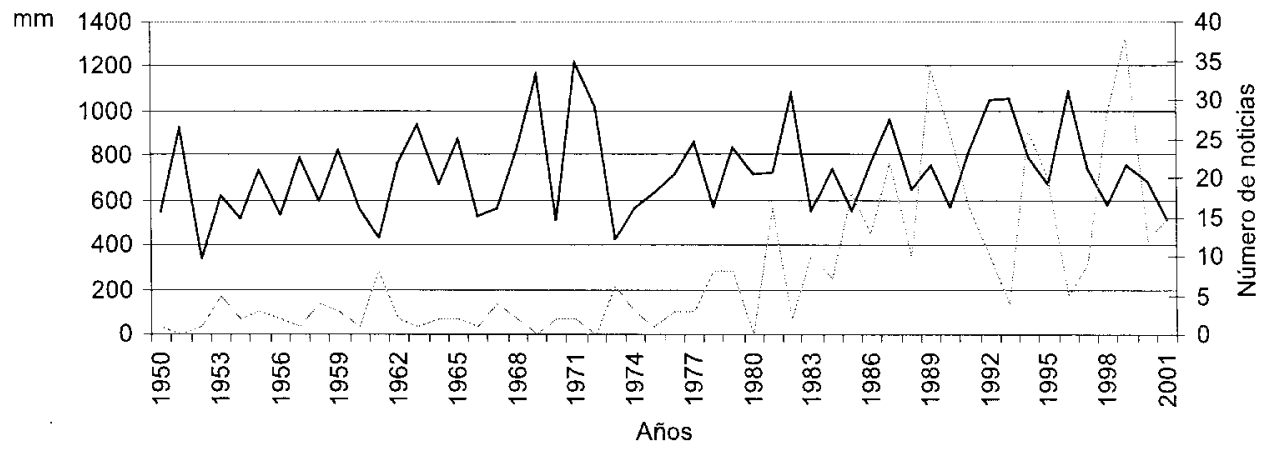

- Precipitación media anual Número noticias

Figura 1. Número de noticias publicadas y precipitación media anual (1950-2001). Fuente: Elaboración propia.

Si el análisis lo realizamos a partir de las secuencias secas (tabla 4), determinadas en el capítulo anterior mediante el cálculo del Índice Estandarizado de Sequías Pluviométricas, observamos que, en general, existe una buena correspondencia entre los periodos climáticos secos y su frecuencia de aparición en la prensa: el $74 \%$ de las noticias que tratan aspectos referentes a las sequías se incluyen en los periodos secos identificados. Por el contrario, el $26 \%$ restante aparece en la prensa coincidiendo especialmente con secuencias secas de corta duración y elevada intensidad, suficientes para agotar las fuentes de suministro y afectar las disponibilidades hídricas. Sería por ejemplo el caso de la ya comentada sequía del verano de 1994 o la que afectó al conjunto de la cuenca entre septiembre y diciembre de 1981. En este último caso, las generosas lluvias de la primavera anterior suavizaron un déficit hidrológico acumulado del año 1980, pero no fueron suficientes para impedir la sequía durante los últimos meses del año 1981. Por suerte, los meses de enero, febrero y marzo del 1982 fueron muy lluviosos en el conjunto de la cuenca, especialmente en la cuenca alta (Darnius, $633 \mathrm{~mm}$ en los tres meses; Figueres, $477 \mathrm{~mm}$; Castelló d'Empúries, $364 \mathrm{~mm}$ ), corrigiendo así los déficits acumulados.

Podemos comprobar (ver tablas 3 y 4) que la tendencia decreciente de la intensidad de las secuencias secas -especialmente en la cuenca alta- no coincide con el aumento registrado de noticias. Este hecho se explica por la dependencia creciente de la disponibilidad de agua en la cuenca a la regulación de sus aguas desde el embalse de Boadella-Darnius $-y$, por lo tanto, a la precipitación en su cabecera-. Podemos intuir la necesidad de umbrales menores de déficit de lluvias para empezar a detectar impactos severos sobre el territorio. Las reducciones pluviométricas entre enero de 1998 y diciembre de 1998, y entre diciembre de 1999 y noviembre del 2000 
Tabla 4. Número total de noticias tratadas en la prensa durante las secuencias secas.

\begin{tabular}{|lc|}
\hline Secuencias secas & Número total de noticias \\
\hline Noviembre 1951-Noviembre 1958 & 18 \\
Noviembre 1959-Septiembre 1962 & 9 \\
Noviembre 1965-Octubre 1968 & 7 \\
Noviembre 1969-Junio 1971 & 2 \\
Octubre 1972-Junio 1977 & 14 \\
Septiembre 1977-Diciembre 1978 & 10 \\
Febrero 1979-Abril 1980 & 6 \\
Diciembre 1982- Enero 1986 & 37 \\
Mayo 1986-Junio 1987 & 16 \\
Junio 1988-Febrero 1992 & 84 \\
Noviembre 1994- Noviembre 1995 & 19 \\
Octubre 1997-Diciembre 2001 & 94 \\
Total & 316 \\
\hline
\end{tabular}

Fuente: Elaboración propia.

en los tramos altos de la cuenca no superan el índice $-1,5$ de intensidad, que equivalen a un total de 94 noticias relacionadas con las características y implicaciones territoriales del evento físico. Además coincide con un periodo lluvioso en la llanura, que podría hacernos pensar en una menor exigencia hídrica de los regadíos durante el conjunto de la temporada de riego.

\section{Conexión espacial entre el evento climático y los impactos territoriales}

Las variaciones en el comportamiento espacial y temporal de las precipitaciones, los distintos sistemas de abastecimiento de agua dispuestos en el territorio y los requerimientos hídricos sectoriales, determinan un grado de dispersión y difusión espacial de los impactos territoriales de la sequía en la cuenca, divergentes incluso entre áreas próximas con unas conductas pluviométricas similares. La prensa refleja con exactitud estas realidades territoriales y demuestra que, en definitiva, los impactos no tienen por qué coincidir espacialmente con las áreas afectadas por el evento físico, especialmente cuando aumentan las posibilidades de regulación del agua disponible.

El análisis de la prensa también permite constatar cómo el aumento de la flexibilización de la oferta hídrica como consecuencia del incremento de la capacidad de regularización de las aguas superficiales en la cuenca, explica que los impactos de una sequía en un espacio determinado no estén condicionados por la precipitación caída en el mismo lugar sino por los resultados pluviométricos en las áreas manan- 
Tabla 5. Número de noticias sobre las implicaciones pluviométricas y/o hidrológicas en los episodios de escasez hídrica en la cuenca del río Muga.

\begin{tabular}{|c|c|c|c|}
\hline \multirow[b]{2}{*}{ Secuencias secas } & \multirow{2}{*}{$\begin{array}{c}\text { Área afectada } \\
\text { por la falta de lluvias }\end{array}$} & \multicolumn{2}{|c|}{ Número de noticias } \\
\hline & & $\begin{array}{c}\text { Déficit de agua } \\
\text { regulada }\end{array}$ & Déficit de lluvias \\
\hline Noviembre 1951-Noviembre 1958 & Cuenca media & 9 & 1 \\
\hline Abril 1952- Febrero 1954 & Guenca baja & 4 & 0 \\
\hline Noviembre 1959-Septiembre 1962 & Cuenca media & 1 & 0 \\
\hline Noviembre 1965-Octubre 1968 & Cuenca baja & 3 & 1 \\
\hline Noviembre 1965-Enero 1967 & Cuenca media & 1 & 0 \\
\hline Noviembre 1969-Febrero 1971 & Cuenca alta & 0 & 1 \\
\hline Diciembre 1969-Junio 1971 & Cuenca mediá & 0 & 1 \\
\hline Octubre 1972-Junio 1977 & Cuenca alta & 1 & 1 \\
\hline Noviembre 1972-Junio 1975 & Cuenca baja & 1 & 0 \\
\hline Septiembre 1977-Diciembre 1978 & Cuenca alta & 2 & 1 \\
\hline Febrero 1979-Abril 1980 & Toda là cuenca & 0 & 0 \\
\hline Diciembre 1982-Abril 1985 & Cuenca baja & 8 & 6 \\
\hline Enero 1983-Octubre 1984 & Cuenca alta & 8 & 3 \\
\hline Abril 1983-Abril 1984 & Cuenca media & 6 & 3 \\
\hline Diciembre 1984-Enero 1986 & Cuenca alta & 0 & 5 \\
\hline Mayo 1986-Junio 1987 & Cuenca alta & 0 & 1 \\
\hline Junio 1988-Diciembre 1991 & Cuenca baja & 4 & 11 \\
\hline Junio 1988-Junio 1990 & Cuenca media & 3 & 6 \\
\hline Septiembre 1988-Octubre 1989 & Cuenca alta & 1 & 5 \\
\hline Diciembre 1989-Febrero 1992 & Cuenca media & 2 & 5 \\
\hline Noviembre 1994- Noviembre 1995 & Toda la cuenca & 3 & 1 \\
\hline Mayo 1998-Junio 1999 & Cuenca baja & 3 & 9 \\
\hline Enero 1998-Septiembre 1999 & Cuenca meclia & 5 & 13 \\
\hline Octubre 1997-Diciembre 2001 & Cuenca alta & 6 & 15 \\
\hline Diciembre 1999 -Diciembre 2000 & Cuenca baja & 1 & 2 \\
\hline Diciembre 1999-Noviembre 2000 & Cuenca alta & 1 & 2 \\
\hline
\end{tabular}

Fuente: Elaboración propia.

tiales. La organización de los aprovechamientos sectoriales del agua en la cuenca dependen en gran medida del estado de las reservas del embalse de BoadellaDarnius, responsable del abastecimiento urbano de la ciudad de Figueres, de algunos núcleos turísticos de la Costa Brava Norte (Llançà, Cadaqués, Roses y Empuriabrava) y del regadio de más de 6.500 hectáreas de la cuenca media y baja del río Muga.

Los resultados de la tabla 5 ponen en evidencia la variación que ha experimentado el grado de dependencia de la sociedad respecto al comportamiento de la precipitación. Mientras que durante los años cincuenta y sesenta las exigencias hidricas de las actividades productivas, especialmente la agricultura (Ribas et al., 1996), dependen estrechamente del ritmo de las precipitaciones (como así lo confirma el elevado número de noticias que aparecen en estos años referentes a los problemas que oca- 
siona a la agricultura la escasez de lluvias), la entrada en funcionamiento del embalse de Boadella-Darnius en 1968 y los importantes cambios que se dan en las formas de organización del territorio (Ribas y Saurí, 2002) implican un cambio trascendental en las formas y sistemas de organización de las necesidades hídricas de los distintos sectores sociales y económicos. A partir de este momento buena parte de la sociedad altoampurdanesa estará más pendiente de las disponibilidades hidrológicas de la presa que no del ritmo de las precipitaciones. Así lo delata la disminución que experimenta a partir de estos años el número de noticias referentes a la fálta de precipitaciones y el aumento de las referentes al nivel de las reservas de agua en el embalse. La entrada en funcionamiento del embalse de Boadella-Darnius permite aumentar el margen de seguridad en la disponibilidad de agua, especialmente en verano, cuando las demandas hídricas agrarias y urbano-turísticas son mayores. De esta forma, en los sectores abastecidos a partir de aguas reguladas, existe cada vez más una estrecha independencia espacial y temporal del ritmo de las precipitaciones locales. En cambio, las actividades vinculadas a la disponibilidad de agua de lluvia de la cuenca alta continuarán mostrándose muy sensibles a los déficits pluviométricos locales.

Todos estos cambios socioterritoriales explican que, a partir de los años setenta, la mayoría de noticias publicadas hagan referencia a la preocupación social por la disminución de los niveles hidrológicos del pantano. Preocupación que irá en aumento, especialmente durante la década de los noventa, cuando se incrementa la dependencia de los diversos sectores sociales y económicos de la cuenca media y baja respecto las disponibilidades hídricas del embalse.

Estos cambios son especialmente visibles cuando ponemos en relación la media anual de agua almacenada en la presa de Boadella-Darnius y el número de noticias publicadas. El umbral para determinar el carácter hidrológico de la presa es la media anual del porcentaje de su capacidad, establecida en el 60,2\% de su capacidad. En concreto se detectan 13 años hidrológicos por debajo de este umbral, con intensidades dispares: desde el 59,6\% del año hidrológico 1973-74 hasta el 24,7\% del año 199899, momento en que alcanza el mínimo histórico de capacidad (el 27 de noviembre de 1998 el embalse sólo cuenta con $8,42 \mathrm{Hm}^{3}$ de agua, lo que equivale al 13,6\% de su capacidad). La prensa recoge con rigor estos episodios más críticos coincidiendo el mayor número de noticias con los meses en que el nivel de las reservas es más bajo (octubre de 1983, julio, agosto, septiembre y noviembre de 1998, y febrero y mayo de 1999) y, especialmente, cuando éstas se traducen en efectos territoriales que, gradualmente, afectan a distintos sectores sociales y económicos de la cuenca. Los meses de verano son, lógicamente, los más sensibles al retroceso de las reservas del embalse debido a la reducción natural de las precipitaciones, el incremento medio de las temperaturas y el aumento de las demandas agrarias y urbano-turisticas.

La importancia de la creciente dependencia de la sociedad del estado de las reservas del embalse de Boadella-Darnius queda reflejada en los resultados de la correlación entre el número de noticias que se refieren a las sequías y la media anual de agua 


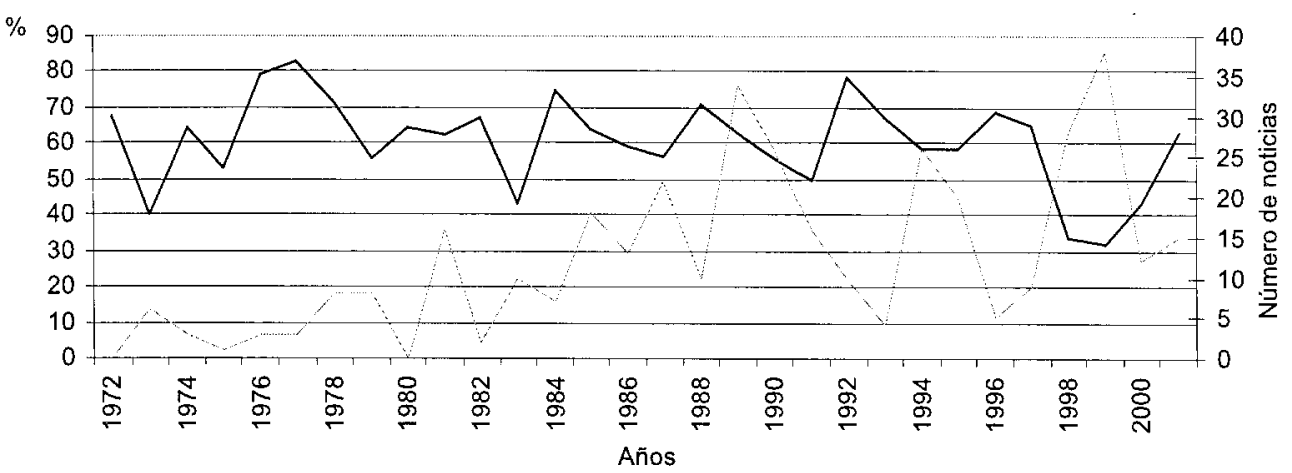

- Agua almacenada Número noticias

Figura 2. Media anual de agua almacenada en la presa de Boadella-Darnius y número de noticias publicadas (1972-2001). Fuente: Elaboración propia.

embalsada, situada en el $-0,56$, dos puntos superior a la relación del noticiario con las manifestaciones pluviométricas $(-0,31)$ en la serie temporal 1972-2001. Con esta medida confirmamos la estrecha vinculación del desarrollo socioeconómico del territorio con las regulaciones superficiales del agua en la cuenca, una dependencia creciente como muestra la evolución de los resultados del cálculo seleccionado, con una relación más estrecha en la década de los años noventa $(-0,74)$ respecto a los ochenta $(-0,49)$, ambos valores superiores a los relativos al comportamiento de la precipitación en la cuenca.

\section{Análisis retrospectivo de los usos, los problemas y las soluciones en la gestión del agua}

Otra de las aplicaciones del análisis temático del contenido de las noticias publicadas consiste en identificar las situaciones de conflicto entre disponibilidad y usos del agua, calidad del agua y de los ecosistemas fluviales e interpretar las soluciones de planificación y gestión del recurso llevado a cabo con el objetivo de resolver estos conflictos.

El conjunto de las 422 noticias publicadas se han agrupado en seis ámbitos temáticos:

- Abastecimiento de agua, que incluye todas aquellas noticias referidas a la denuncia de limitaciones estructurales de abastecimiento de agua para distintos usos y sectores, obras y proyectos de mejora o ampliación del suministro 
de agua, principalmente para usos urbanos y agrarios, y las diversas respuestas sociales e institucionales a los episodios de sequí;

- Saneamiento básico, con noticias relativas a las limitaciones estructurales de las obras de saneamiento básico y a las actuaciones y proyectos de obras de saneamiento de aguas residuales;

- Impacto ambiental, que incluye los aspectos relacionados con el estado ambiental del medio fluvial, como la propuesta de programas para la recuperación de los recursos hídricos, las evaluaciones del riesgo de incendios forestales como indicadores de la sensibilidad de la cuenca a los procesos naturales de la sequía o la extensión de la degradación ambiental en los ambientes fluviales de la cuenca;

- Contaminación, que precisa los episodios recurrentes de contaminación de las aguas del río Muga por vertidos generalmente industriales y por la escasez de procesos de depuración de las aguas residuales urbanas. Se recogen asimismo las noticias referidas a los problemas de salinización de los acuíferos y al riesgo que ello supone para la garantía de la cantidad y la calidad de los abastecimientos hídricos;

- Estado hidrológico, que se hace eco del estado de las reservas hídricas superficiales (embalse de Boadella-Darnius) y subterráneas en la cuenca, así como del estado de los caudales de los ríos como indicadores cuantitativos de la escasez de agua;

- Meteorología extrema, que incluye los episodios de lluvia abundante que suelen atenuar o anular el avance de los periodos climáticos secos, así como las noticias referidas directamente a la disminución de las precipitaciones y sus efectos sobre los cultivos especialmente dependientes del ritmo de las precipitaciones, los temporales de viento fuerte y seco del norte que anulan en muchas ocasiones los efectos beneficiosos de los episodios de precipitaciones precedentes.

Tabla 6. Clasificación de las noticias según ámbitos temáticos.

\begin{tabular}{|lcc|}
\hline Ámbito temático & Número de noticias & $\%$ \\
\hline Abastecimiento de aguá & 168 & 40 \\
Saneamiento básico & 53 & 13 \\
Impacto anbiental & 43 & 10 \\
Contaminación & 29 & 7 \\
Estado hidrológico & 52 & 12 \\
Meteorología extrema & 77 & 18 \\
Total & $\mathbf{4 2 2}$ & $\mathbf{1 0 0}$ \\
\hline
\end{tabular}

Fuente: Elaboración propia. 
Tabla 7. Número de noticias según temas y subtemas.

\begin{tabular}{|lcccccccc|}
\hline & $\begin{array}{c}\text { Abastecimiento } \\
\text { agua }\end{array}$ & $\begin{array}{c}\text { Saneamiento } \\
\text { básico }\end{array}$ & $\begin{array}{c}\text { Impacto } \\
\text { ambiental }\end{array}$ & $\begin{array}{c}\text { Contaminación } \\
\text { Estado } \\
\text { hidrológico }\end{array}$ & $\begin{array}{c}\text { Meteorología } \\
\text { extrema }\end{array}$ & Total \\
\hline Obras & 58 & 25 & 0 & 0 & 0 & 0 & 83 \\
Degradación ambiental & 0 & 0 & 24 & 8 & 0 & 0 & 32 \\
Proyectos & 28 & 13 & 4 & 0 & 0 & 0 & 45 \\
Déficit agricola & 3 & 0 & 0 & 0 & 0 & 27 & 29 \\
Déficit urbano & 17 & 0 & 0 & 0 & 10 & 0 & 26 \\
Planificación & 0 & 0 & 2 & 0 & 0 & 0 & 2 \\
Programa & 20 & 2 & 4 & 0 & 0 & 0 & 26 \\
Calidad agua & 0 & 0 & 0 & 21 & 0 & 0 & 21 \\
Alerta restricciones & 15 & 0 & 0 & 0 & 0 & 0 & 15 \\
Lluvias abundantes & 5 & 0 & 0 & 0 & 0 & 23 & 28 \\
Déficit técnico & 18 & 13 & 0 & 0 & 0 & 0 & 31 \\
Déficit lluvias & 0 & 0 & 0 & 0 & 0 & 27 & 27 \\
Déficit fluvial & 0 & 0 & 9 & 0 & 42 & 0 & 51 \\
Ayudas económicas & 4 & 0 & 0 & 0 & 0 & 0 & 4 \\
Total & 168 & 53 & 43 & 29 & 52 & 77 & $\mathbf{4 2 2}$ \\
\hline
\end{tabular}

Fuente: Elaboración propia.

El ámbito temático que reúne un mayor número de noticias corresponde al abastecimiento del agua (168 noticias, el 40\% del total), seguido de las noticias relacionadas con las conductas extremas de la precipitación ( 77 noticias, 18\% del total), principalmente con la escasez de las lluvias, así como la actividad de los episodios protagonizados por el fuerte viento de tramontana. Les siguen las referencias a los déficits de saneamiento del agua $(53,13 \%)$, el ámbito del estado hidrológico $(52,12 \%)$, las repercusiones ambientales de la sequía $(43,10 \%)$ y los episodios de contaminación de los ríos y acuíferos de la cuenca $(29,7 \%)$. Se han propuesto diferentes subtemas (ver tabla 7) a fin de concretar la caracterización de las categorías principales.

Entre las noticias correspondientes al ámbito abastecimiento de agua, la mayoría de registros se concentran en: a) obras de captación y distribución del agua para abastecimientos agrarios y, especialmente, urbanos (34\%); b) proyectos que plantean medidas estructurales para el suministro del agua $(17 \%)$; c) medidas coyunturales de ajuste a los déficits mediante programas municipales de ahorro de agua para usos públicos y domésticos (12\%) y; d) los déficits de agua para usos urbanos (10\%) y agrícolas (2\%). En relación al ámbito meteorología extrema, los tres subtemas comparten porcentajes similares, con un 35\% las temáticas relacionados con los déficits de agua para usos agrícolas (preferentemente en las tierras de secano), un 30\% relacionado con los episodios de lluvia abundante (suficientes en muchos casos para el cese de la sequía), y también el 35\% referidos estrictamente a la escasez de precipitaciones. El ámbito saneamiento básico incluye referencias a las infraestructuras de depuración de aguas residuales ( $46 \%$ ), a los déficits técnicos que agravan la contaminación de los ríos, reducen la disponibilidad del agua y alteran el equilibrio de los ecosistemas fluviales (25\%), a 
los proyectos y estudios de la necesidad de las actuaciones de depuración (25\%), y a la propuesta de planes integrales de mejora y saneamiento de las riberas del río Muga (4\%). Las consecuencias ambientales de las actuaciones humanas sobre los sistemas naturales suponen el $56 \%$ de las noticias referidas al tema impactos ambientales, preocupadas por la alteración de los ecosistemas fluviales y el $21 \%$ al déficit de caudal ecológico. El tema de contaminación se refiere en un 29\% a las noticias relacionadas con la contaminación de las aguas subterráneas, y en un $71 \%$ a los episodios de contaminación de los ríos. En relación al estado bidrológico de la cuenca las noticias se agrupan en un $81 \%$ en el subtema de la regresión de los niveles hidrológicos superficiales, y en un 19\% en el apartado que expresa la reducción de la disponibilidad de los acuíferos que abastecen principalmente núcleos urbanos.

La agrupación de la serie de noticias en intervalos de cinco años nos permite analizar cronológicamente el significado de las informaciones temáticas y su relación con los subtemas.

Varios son los factores que explican la evolución que han tenido las temáticas relacionadas. Por una parte, la propia evolución que ha experimentado el acceso y el tratamiento de la información dada por los medios de comunicación. Por otra parte, las limitaciones técnicas de abastecimiento y depuración del agua debido al aumento progresivo de la población y las demandas equivalentes (significativos durante la segunda mitad de los años ochenta). También, la mayor sensibilización mostrada por la prensa hacia los aspectos ambientales, y al aumento de la vulnerabilidad de la cuenca a la escasez de agua ante déficits menores de precipitación -ésta muy vinculada durante los años noventa al ritmo de la recarga de los niveles del pantano de Boadella-Darnius-. Por último, la mayor sensibilidad a reducciones pluviométricas estacionales que recortan la funcionalidad de la regularización del embalse.

Tabla 8. Evolución cronológica del número de noticias según ámbitos temáticos.

\begin{tabular}{|lccccccc|}
\hline $\begin{array}{c}\text { Abastecimiento } \\
\text { de agua }\end{array}$ & $\begin{array}{c}\text { Saneamiento } \\
\text { Básico }\end{array}$ & $\begin{array}{c}\text { Impacto } \\
\text { ambiental }\end{array}$ & $\begin{array}{c}\text { Contaminación } \\
\text { Estado } \\
\text { hidrológico }\end{array}$ & $\begin{array}{c}\text { Meteorologia } \\
\text { extrema }\end{array}$ & Total \\
\hline $\mathbf{1 9 5 1 - 1 9 5 5}$ & 3 & 0 & 0 & 0 & 2 & 7 & 12 \\
$\mathbf{1 9 5 6 - 1 9 6 0}$ & 3 & 0 & 0 & 0 & 0 & 8 & 11 \\
$\mathbf{1 9 6 1 - 1 9 6 5}$ & 9 & 0 & 1 & 0 & 0 & 6 & 16 \\
$\mathbf{1 9 6 6 - 1 9 7 0}$ & 0 & 1 & 0 & 1 & 2 & 4 & 8 \\
$\mathbf{1 9 7 1 - 1 9 7 5}$ & 6 & 3 & 1 & 0 & 1 & 1 & 12 \\
$\mathbf{1 9 7 6 - 1 9 8 0}$ & 7 & 5 & 1 & 4 & 1 & 4 & 22 \\
$\mathbf{1 9 8 1 - 1 9 8 5}$ & 12 & 2 & 0 & 9 & 9 & 21 & 53 \\
$\mathbf{1 9 8 6 - 1 9 9 0}$ & 43 & 20 & 13 & 10 & 12 & 7 & 105 \\
$\mathbf{1 9 9 1 - 1 9 9 5}$ & 35 & 16 & 6 & 2 & 10 & 8 & 77 \\
$\mathbf{1 9 9 6 - 2 0 0 0}$ & 42 & 6 & 18 & 1 & 14 & 10 & 91 \\
Total & 160 & 53 & 40 & 27 & 51 & 76 & $407^{*}$ \\
\hline
\end{tabular}

* Contabilizado hasta el año 2000 para mantener intervalos regulares y así facilitar la comparación. El año 2001 suma un total de 15 noticias.

Fuente: Elaboración propia. 
La tabla 8 permite la lectura de la distribución temporal de las noticias referidas a la escasez del agua en la cuenca, con la mayor frecuencia de registros en la segunda mitad de los años ochenta, seguido de los años noventa. El menor número de noticias se concentra entre los años 1966 y 1970 , y 1956 y 1960 . El aumento de noticias que se refieren al abastecimiento tiene lugar paralelamente al crecimiento demográfico y urbanístico de la cuenca y el consecuente aumento de los consumos de agua, que pone en evidencia la insuficiencia de la infraestructura hidráulica existente para dar respuesta a las demandas que se generan. Durante la segunda mitad de los años ochenta y primera de los noventa se toman las mayores decisiones para afrontar los problemas de saneamiento de las aguas del río Muga con la construcción y entrada en funcionamiento de depuradoras y potabilizadoras públicas para garantizar la calidad del recurso. Es entre el año 1986 y 1990 cuando se registran los casos más importantes y reincidentes de contaminación fluvial, con repetidas confrontaciones entre los ayuntamientos de Figueres y Castelló d'Empúries, los municipios máximos responsables del mal estado ecológico que presenta en este periodo el río Muga. También a finales de los años noventa, la falta de precipitaciones se traduce en la falta de agua circulante en el río Muga, sus afluentes y en los humedales litorales. El periodo 1981-1985 recoge 21 casos de fenómenos pluviométricos extremos, con acusados déficits de lluvia en los años 1983 y 1984 que afectaron de forma importante a la producción de los cultivos de invierno. La persistencia de esta secuencia seca causó importantes incertidumbres entorno a la rentabilidad de las cosechas y mucha preocupación en el campo, ampliamente recogida en la prensa. La alarma se repite en los años 1999 y 2000 cuando se generalizan las restricciones de agua para el riego. La campaña de 1999 es la primera desde la entrada en funcionamiento del plan de regadíos del río Muga en la que no se pueden satisfacer las necesidades hídricas de los cultivos por el bajo nivel que presentaban las reservas de agua en el pantano de Boadella-Darnius.

La ampliación de los sistemas de suministro de agua potable en los núcleos turísticos de la Costa Brava Norte durante los años setenta coincide con los episodios más intensos de contaminación de acuíferos litorales, lo que obliga a buscar nuevas fuentes de suministro de agua lejos de las locales. De ello se hace especial eco la prensa durante las tres últimas décadas con la proliferación de noticias referidas al aumento de los niveles de contaminación del agua, el aumento de las tensiones y conflictividades sociales ante los déficits hídricos, las menores disposiciones hidrológicas de la presa de Boadella-Darnius, etc.

Por municipios (ver mapa 2), las ciudades de Figueres y Castelló d'Empúries reúnen los porcentajes de noticias más altos principalmente por su peso demográfico y las implicaciones que supone el déficit hídrico en el abastecimiento de poblaciones. Los núcleos urbano-turísticos de Roses, Llançà, Cadaqués también 


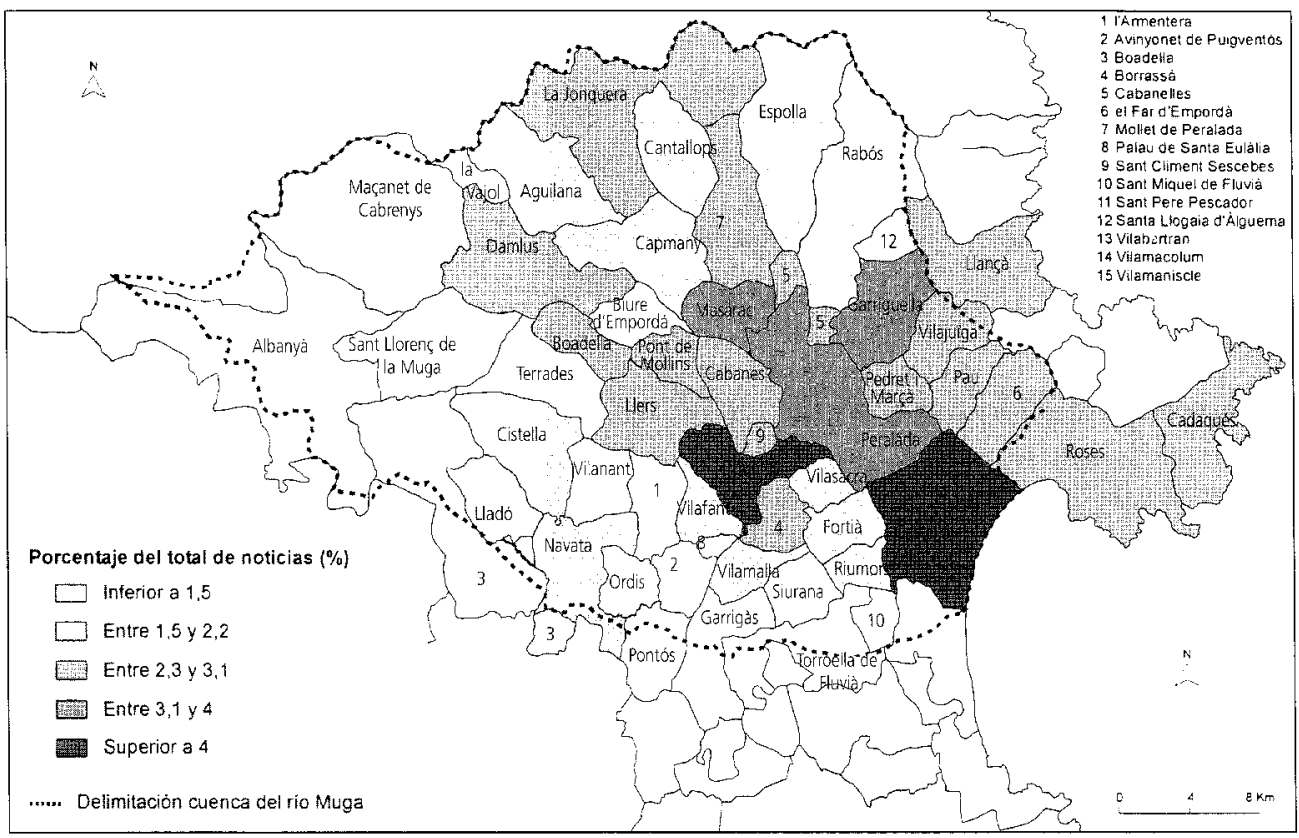

Mapa 2. Regionalización municipal de las noticias publicadas en L’Empordà.

concentran un buen número de noticias, mayoritariamente asociadas a los problemas que ocasiona el abastecimiento del agua potable para usos esencialmente urbanos y recreativos. Se distinguen asimismo porcentajes altos en las principales áreas de influencia de Figueres, la capital de la comarca, resultado de las insuficiencias infraestructurales en el suministro del agua potable ante el crecimiento urbano de estos núcleos durante los años ochenta y noventa, que en muchos casos requieren la revisión de sus fuentes de abastecimiento y tienden a una adhesión a las disponibilidades del embalse de Boadella-Darnius (serían los casos, por ejemplo, de los municipios de Llers y Cabanes). Igualmente sucede con los pueblos de la Serra de l'Albera (Sant Climent Sescebes, Masarac, La Jonquera, Espolla y Capmany), que se conectan al acuífero de Peralada. En los municipios litorales también se registran un buen número de noticias relativas a la degradación del estado ambiental de los ambientes fluviales, así como la incidencia del riesgo frecuente de incendios forestales a los sectores forestales de la cuenca. Asimismo destacan de forma significativa los municipios de la cuenca media y baja de clara vocación agrícola, siempre pendientes de las oscilaciones en el comportamiento de las precipitaciones y de las reservas hidrológicas del embalse de Boadella-Darnius. 


\section{Conclusiones}

El análisis del tratamiento de las noticias de prensa nos permite evaluar la evolución de la dependencia de la sociedad respecto al ritmo de las precipitaciones, ampliamente referenciado en apartados anteriores, y necesario para entender los estados de la conmoción social a la problemática de la escasez del agua. En los años cincuenta la sequía es, en la cuenca del río Muga, un fenómeno cotidiano, una norma asumida por una sociedad que sitúa el umbral de sequía en consonancia a la recurrencia de las reducciones pluviométricas.

El aumento progresivo de la flexibilización de la oferta del agua permite incorporar nuevas posibilidades de intervenir en las disponibilidades hídricas, preferentemente en la agricultura de regadío, antes expuesta a la frecuencia estricta de las lluvias, mientras que a partir de los años setenta pueden garantizarse en las áreas abastecidas por aguas reguladas resultados más eficientes, con la previsión de crisis hidrológicas que pueden flexibilizar la demanda (cambio de cultivos) y adaptarse a las condiciones de sequía.

En los años setenta, las noticias publicadas en L'Empordà son reflejo de los desequilibrios entre la insuficiencia técnica y el crecimiento desmesurado de las demandas hídricas producto de un modelo territorial donde conviven una agricultura cada vez más intensiva con un proceso urbanizador que parece no tener fin. Ya no es la misma base de la sociedad la que se va a conmover ante los déficits pluviométricos; ahora saltan a la prensa las incomodidades derivadas de la escasez de agua más que los problemas productivos que ésta genera (Pita López, 1989). En este sentido, la sequía se convierte gradualmente en un fenómeno urbano. La mayor capacidad tecnológica y financiera favorece la demanda social de más exigencia en los aprovechamientos del agua y más responsabilidad administrativa ante las repercusiones económicas derivadas de una secuencia seca.

A partir de finales de la década de los setenta, el incremento de la sensibilidad ambiental explica también la evolución del carácter de las sequías. Surgen más movimientos conservacionistas y se elaboran programas de defensa y conservación del medio para poder atender de forma sostenible las demandas de agua con el mayor respeto a las cualidades ambientales de la cuenca. Una rígida reivindicación social, apoyada por todos los sectores implicados en el uso del agua, consiguió el reconocimiento de una extensa zona de humedales como parque natural en el Alt Empordà. La prensa refleja la emergente sensibilización ambiental de la sociedad mediante el tratamiento de noticias relacionadas con el medio ambiente, especialmente hídrico. El $92 \%$ de estas noticias se recogen entre los años ochenta y sobre todo noventa. 
También la mayor frecuencia de la conflictividad social en el ámbito de estudio durante los años ochenta y noventa ponen en evidencia la creciente vulnerabilidad de la cuenca a la escasez del agua, expresada en el marco de políticas hidráulicas que fomentan el incremento de la oferta del agua y poca atención prestan a la incidencia en las demandas hídricas. La publicación creciente de noticias que se refieren a los problemas reincidentes en el abastecimiento del agua, a los retrocesos constantes de los niveles de agua del embalse y las referencias directas a las necesidades de precipitaciones inmediatas, son indicadores de la propia recurrencia natural de las manifestaciones irregulares de las precipitaciones pero, sobre todo, de la falta de una planificación de territorio y sus recursos que tenga en la cuenca fluvial su ámbito de aplicación. La prensa regional ha resultado ser una valiosa herramienta analítica y de diagnóstico para reconocer la incidencia histórica y actual de las sequías en la cuenca, y, a partir de aquí, plantear formas de planificación y gestión del agua acordes a las necesidades sociales y territoriales de cada ámbito de estudio.

\section{Agradecimientos}

Este artículo se inscribe en el proyecto de investigación "Estudio integrado del ciclo del agua: el caso de la cuenca del río Muga (Girona)" concedido por el Ministerio de Ciencia y Tecnología (REN2000-0453-Co2-02/HID). Los autores de este trabajo quieren agradecer a los observadores meteorológicos y a los organismos públicos competentes la información facilitada para la realización de este trabajo.

\section{Notas}

$1 \mathrm{El}$ ámbito de estudio agıupa los límites estrictamente naturales de la cuenca hidrográfica del río Muga, además de los municipios de Llançà, Cadaqués y Roses, por depender su abastecimiento urbano de las aguas reguladas de la cuenca del río Muga.

2 Un año se considerará muy seco cuando la precipitación registrada corresponda al $20 \%$ de los años más secos, y será seco cuando la precipitación observada sea superior o igual al $20 \%$ de los años más secos e inferior al $40 \%$ de los más secos. Esta lógica considera que los años normales, húmedos y muy húmedos se dan cuando las precipitaciones están comprendidas en el intervalo del 40 al $60 \%$, del 60 al $80 \%$, y superior al $80 \%$, respectivamente.

3 La precipitación media anual ha sido calculada a partir de la media de una muestra regular de alta representatividad espacial y temporal de observatorios meteorológicos por tramos de la cuenca. 


\section{Bibliografía}

Almarza, C. (2000): "Sequías: Indicadores de alerta, intensidad y seguimiento. Análisis espacio temporal". En Llamas, M.R. (ed.): "Aguas subterráneas y sequías". Revista de la Real Academia de Ciencias Exactas, Físicas y Naturales, vol. 94.

Bayés Bruñol, C. (2002): Caracterització geográfica de les sequeres a la conca del riu Muga (1950-2001). Gerona, Universidad de Gerona. Memoria de investigación del programa de Doctorado en Medio Ambiente.

Compte, A. (1968): "Tipus de temps a l'Empordà". Annals de l'Institut d'Estudis Empordanesos, 7, p. 135-167.

Gil Olcina, A. y Morales, A. (coords.): Causas y consecuencias de las sequias en España. Alicante, Caja de Ahorros del Mediterráneo e Instituto Universitario de Geografía.

Llamas, M.R. (2000): "Aguas subterráneas y sequías". Revista de la Real Academia de Ciencias Exactas, Físicas y Naturales, vol. 94, 2, p. 147-157.

Martín Vide, F.J. (1990): "Diez características de la pluviometría española decisivas en el control de la demanda y el uso del agua". Boletín de la Asociación de Geógrafos Españoles, 18, p. 9-16.

Morales Gil, A., Olcina, J, i Rico, A.M. (1999): "Diferentes percepciones de la sequía en España: adaptación, catastrofismo e intentos de corrección". Investigaciones Geográficas, 22, p. 5-46.

Olcina Cantos, J. (2001): "Causas de las sequías en España. Aspectos climáticos y geográficos de un fenómeno natural". En: Gil Olcina, J, y Morales Gil, A. (eds.).
Causas y consecuencias de las sequias en España. Alicante, Caja de Ahorros del Mediterráneo y Instituto Universitario de Geografía, p. 49-109.

Pita López, M.F. (1985): "El papel de la prensa en el estudio de las sequías". En V.AA., IX Coloquio de Geógrafos Españoles. Murcia, AGE y Sección de Geografia de la Universidad de Murcia.

Pita López, M.F. (1989): "La sequía como desastre natural. Su incidencia en el ámbito español". Norba, 6-7, p. 31-61.

Pita López, M.F. (1990): "Reflexiones entorno a las sequías". Boletín de la Asociación de Geógrafos Españoles, 10, p. 21-39.

Pita López, M.F. (2001): "Un nouvel indice de sécheresse pour les domaines méditerranéens. Application au bassin du Guadalquivir (Sud-ouest de l'Espagne)". En VV.AA., XIII Colloque de L'Association International de Climatologie. Francia.

Pita López, M.F. (2001): "Sequías en la cuenca del Guadalquivir". En: Gil Olcina, J. i Morales Gil, A. (eds.). Causas $y$ consecuencias de las sequias en España. Alicante. Caja de Ahorros del Mediterráneo y Instituto Universitario de Geografía (Universidad de Alicante), p. 310-313.

Ribas, A. y Saurí, D. (ed.) (2002): Canvis socioambientals a l'Alt Empordà. Natura $i$ bistòria en l'evolució recent del paisatge altempordanès. Girona, Càtedra de Geografia i Pensament Territorial i Institut de Medi Ambient de la Universitat de Girona.

Ribas, A., Roset, D., Sanz, C. y Saurí, D. (1996): "Cambios y contradicciones de la 
planificación hidráulica en las cuencas internas de Catalunya: los regadios en la cuenca de La Muga (Girona)". En VV.AA., VIII Coloquio de Geografia Rural. Actas: Comunicaciones. Zaragoza, Departamento de Geografía y Ordenación del Territorio de la Universidad de Zaragoza y Grupo de Trabajo de Geografía Rural (A.G.E.), p. $517-527$.

Ribas, A., Roset, D. y Ventura, M. (1998): “Los cambios de uso del agua en el Alt Empordà (Girona): estrategias de gestión y conflictividad de usos". I Congreso sobre Planificación y Gestión de Aguas. Zaragoza, Universidad de Zaragoza, publicación en $C D$.

Ruiz Urrestarazu, E. (dir.) (1998); El clima del País Vasco a través de la prensa. Grupo de
Climatología de la UPV/EHU. Servicio de Meteorología del Gobiemo Vasco. 212 pp.

Ventura, M., Ribas, A. y Saurí, D. (2000): "Gestión del agua y conflictividad social en la cuenca del río Muga (Alt Empordà)". Geographicalia, 38, p. 59-75.

Silva, F., Pacheco, A.I., Oliveira, E.S., Correia, E.C., Alves, P.C. y Vieira, S. (2002): "O tema da água visto pela impresa regional. O caso do jornal "O Comércio de Guimarâes". En VV.AA., Actas-comunicaciones III Congreso Ibérico sobre Gestión y Planificación del Agua. Sevilla, Universidad de Sevilla, Fundación Nueva Cultura del Agua y Universidad Pablo de Olavide, p. 631-640.

Wilhite, D. (ed.) (2000): Drought. A global Assessment. Londres, Routledge, vol. 2. 